\title{
Healthcare System for Non-invasive Fall Detection in Indoor Environment
}

\author{
Marco Mercuri ${ }^{1}$, Carmine Garripoli ${ }^{1,2}$, Peter Karsmakers ${ }^{1}$, Ping Jack Soh ${ }^{1}$, \\ Guy A. E. Vandenbosch ${ }^{1}$, Calogero Pace ${ }^{2}$, Paul Leroux ${ }^{1}$, Dominique Schreurs ${ }^{1}$ \\ ${ }^{1}$ KU Leuven, Department of Electrical Engineering, Kasteelpark Arenberg 10 bus 2444, \\ 3001 Heverlee (Leuven), Belgium \\ \{Marco.Mercuri, Peter.Karsmakers, PingJack.Soh, \\ Guy.Vandenbosch, Paul.Leroux, Dominique.Schreurs $\} @$ esat.kuleuven.be \\ Garripoli.Carmine@gmail.com \\ ${ }^{2}$ Università della Calabria, Dipartimento di Informatica, Modellistica, Elettronica e \\ Sistemistica, via Pietro Bucci 42/C, 87036 Rende (CS), Italy \\ Calogero.Pace@unical.it
}

\begin{abstract}
Fall incidents and the sustained injuries represent the main causes of accidents for elderly people, and also the third cause of chronic disability. The rapid detection of a fall event can reduce the mortality risk, avoiding also the aggravation of injuries. In this paper an embedded healthcare system based on a microwave radar is presented. A Continuous Wave (CW) Doppler radar is used to detect the changes in speed of different persons experienced during daily activities, namely falling and normal/random movements. The resulted speed signals are then processed in real-time by a digital signal processor (DSP) in order to detect fall incidents. Experimental results, conducted on real human volunteers in a real room setting, have shown a success rate of $100 \%$ in detecting fall events. Moreover, no false positives have been reported.
\end{abstract}

\section{Introduction}

The elderly population has been steadily increasing worldwide. This situation, together with the shortage of nursing homes and the natural desire to stay at home, has resulted in a growing need for healthcare approaches that emphasize routine long-term monitoring in the home environment. Elderly people who live alone are usually exposed to health risks which in some cases may cause fatality. In fact, fall incident among the elderly is considered one of the major problems worldwide, and often result in serious physical and psychological consequences [1]. Research pointed out that $30 \%$ to $45 \%$ of the persons older than 60 years fall at least once a year. People who experience a fall event at home, and remain on the ground for an hour or more, may suffer from many medical complications, such as dehydration, internal bleeding, and cooling, and half of them die within six months [2]. The delay in hospitalization increases mortality risk. Studies have shown that the longer the person lies on the floor, the poorer is the outcome of medical intervention [3], [4]. For that 
reason, it is imperative to detect falls as soon as they occur such that immediate assistance may be provided.

Current health monitoring systems are based on necklace or wristwatch with a button that is activated by the patient in case of an accident. However, in emergency situations, this imposes an important risk factor. In fact, the person may forget to wear the device, or likely may no longer be able to press the button. The ideal solution is therefore a contactless approach that avoids the need for actions by the elderly person. Systems under investigation in the latter category are based on video cameras, floor vibration, and acoustic sensors. In the case of the video camera method, researchers are currently trying to address challenges related to low light, field of view, and image processing, but also privacy is a concern [5]. Floor vibration and acoustic sensors have limited success due to the environmental interference and background noise [6].

Due to the disadvantages of existing fall detection technologies, there is a need for further solutions. An alternative approach based on radar techniques has been demonstrated by the authors [7], [8]. The system uses a machine learning technique to distinguish fall events from normal movements as described in [9].

In this paper an embedded healthcare system based on microwave radar measurements is described. As opposed to [8], a digital signal processor (DSP) platform has been used to process the motioning signals in order to detect fall emergencies in real-time.

The embedded healthcare system and the data processing technique used to process the monitoring signals are introduced in Section 2. The implementation of the data processing technique by means of a DSP is detailed in Section 3. Experimental results are shown in Section 4.

\section{Embedded Healthcare System}

The embedded healthcare system used to design the real-time fall detector has been described by the authors in [8]. It consists of a sensor, combining both radar and wireless communications features, and a base station for data processing (Fig. 1). The sensor integrates a radar module, a Zigbee module, and a microcontroller, while the base station consists of a Zigbee module and the TMS320C6678 DSP platform.

A Continuous Wave $(\mathrm{CW})$ waveform at $5.8 \mathrm{GHz}$ is generated and used to detect the speed signals produced by the test persons during daily activities, namely falling and normal/random movement. The resulting baseband signals are digitized and transmitted to a base station to be processed.

A movement classification based on a Least Squares Support Vector Machines (LS-SVM) approach combined with Global Alignment (GA) kernel [9] is applied to analyze the digitized baseband speed signals in order to distinguish falls from other movements. The technique aims at assessing the changes in speed experienced during a fall or a normal movement. During a fall, in fact, the speed continuously increases until the sudden moment when the movement stops abruptly. During a normal movement, the Doppler signal experiences a controlled movement. More precisely, while a person is sitting down, the speed first gradually increases, and then decreases to a smooth stop, whereas during a walk the speed is quite constant over time. 
The developed algorithm consists of two stages of data analysis, namely the training phase and the testing phase (Fig. 2). Both phases use the digitized speed signal as input.
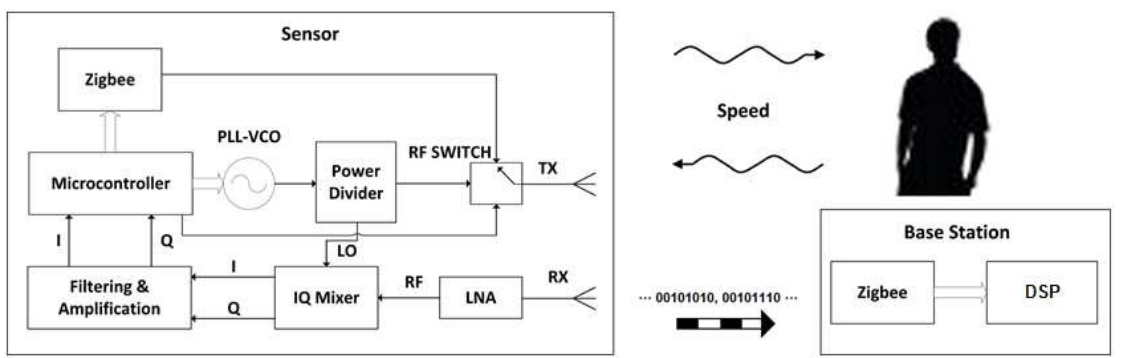

Fig. 1. Simplified block diagram of the embedded healthcare system.

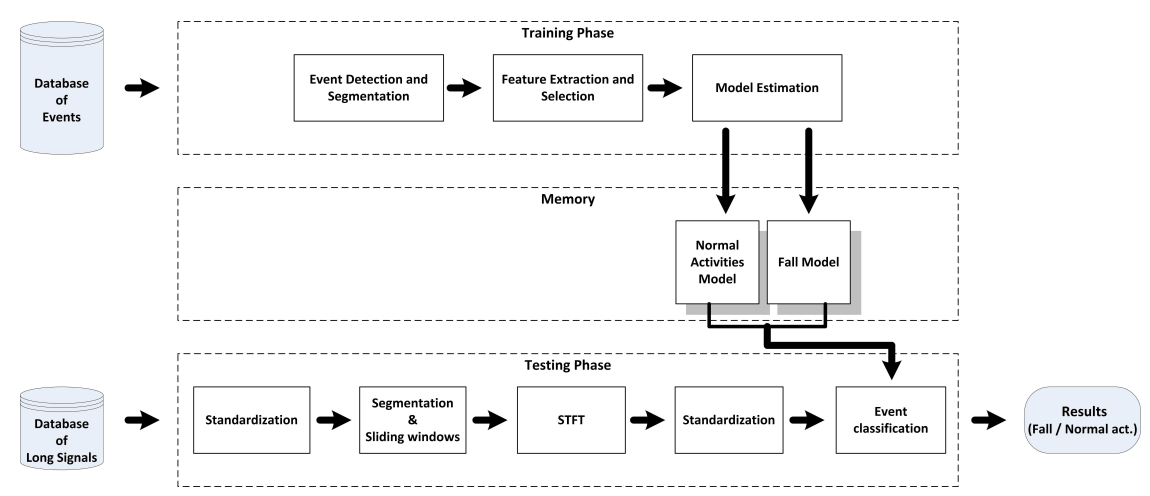

Fig. 2. Block diagram of the implemented classification technique.

\subsection{Training Phase}

The training phase consists of activity detection and segmentation, feature extraction, feature selection, and model estimation. The training activities are divided in two main groups, namely fall events and normal movements. These acquired activities are used to build a data set. However, before learning a model, each activity is grouped in a segment of 2 seconds, considered sufficient to cover the details of the activities and mainly the fall event. This operation of segmentation consists in the detection of the activity energy's peak and in cutting the signal around this peak. Given such segments, the data is preprocessed, namely it is first standardized, such that each dimension has zero mean and unit standard deviation, and then transformed using the Short Time Fast Fourier Transform (STFT) from which only the magnitude spectrum is retained. Prior to the learning phase, the data is again standardized. Once the learning process is finalized, the model is created and stored in a memory to be used in the validation stage. 


\subsection{Testing Phase}

The algorithm performed in this phase presents a structure similar to the data processing of the training phase (Fig. 2). However, the main difference lies in the segmentation stage where the sliding window principle is applied due to the fact that the starting and ending points of the activities are unknown. The size of the sliding window has been fixed to $2 \mathrm{~s}$ in order to be consistent with the length of the activities' segments in the training phase, while the overlap among windows is fixed to $95 \%$.

\section{Classification Implementation}

In order to process a continuous stream of radar signals consisting of multiple activities invoked at unknown instants, the sliding window principle should be applied continuously to the received data. The sensor node transmits to the base station the speed signals every $100 \mathrm{~ms}$. Therefore, every time a new frame is available, the relative samples are concatenated with the last $1.9 \mathrm{sec}$. of the previous signals to create a window of 2 seconds and to have an overlap of $95 \%$. This large overlap is used to improve the performance of the system. In fact, a larger overlap involves a higher number of classifications such that a fall event will be considered over multiple windows, making the system much more immune to the noise that could generate a false positive in a single window classification. Finally, each segment is then preprocessed and classified.

A custom parallelization technique involving the 8 cores of the TMDS320C6678 DSP has been implemented to perform real-time classification. The strict requirement is that a segment classification must be performed before a new Zigbee communication occurs, that means in a time shorter that $100 \mathrm{~ms}$. The pseudo-code of the classification is shown in Fig. 3. The core of the algorithm is to determine the Kernel array $K$, whose elements are calculated by the function computeGAK(Test, Training), that is an off-the-shelf $\mathrm{C}$ code that computes the Global Alignment Kernel for the LS-SVM algorithm. Finally, the function $f(K)$ returns a value that, compared to a threshold, determines whether the segment contains fall or normal movement data. For each iteration of the loop, 8 elements of the Kernel matrix $K$ are computed at the same time by the 8 cores of the DSP. Each core invokes the function computeGAK() independently.

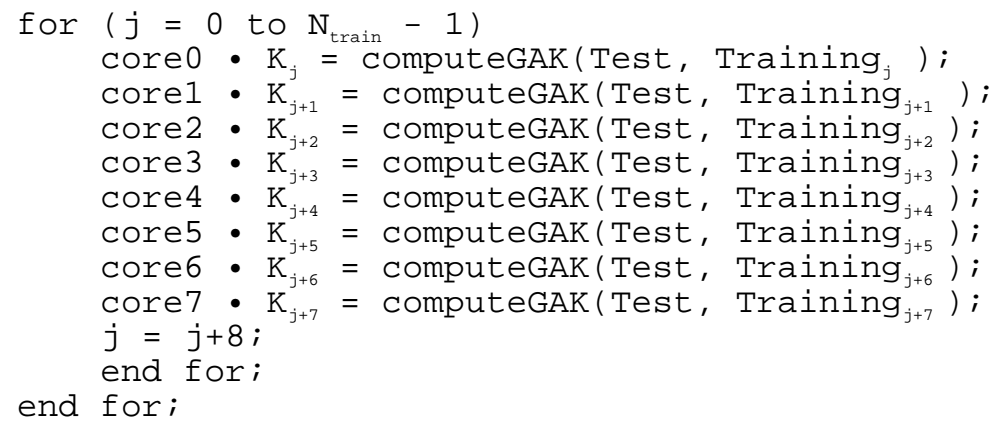




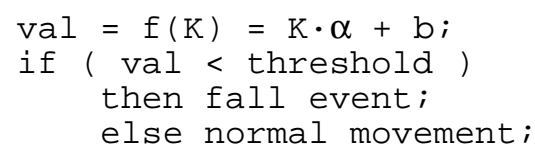

Fig. 3. Classification pseudo-code. $N_{\text {train }}$ represents the number of matrices in the Training structure. Training and Test are thee-dimensional matrices resulting respectively from the model estimation and the preprocessing in the testing phase. The vector $\alpha$ and the constant $b$ are variabiables estimathed in the training phase.

\section{Experimental Results}

A training set containing 80 activities executed by a single test person is used to estimate the activity classification model. The models have consequently been tested for 3 hours on 3 different test persons, for a total of 36 signals of 5 minutes each. Each of these signals was acquired with a single volunteer in the room at a time, and who had not contributed to the training model. The subjects were allowed to mimic typical domestic situations without any restriction in their movements. Moreover, each signal contained only one fall event invoked in a random instant.

The success rate of the system was calculated as the percentage of detected falls. The results have indicated a success rate in detecting fall incidents in real-time of $100 \%$, without reporting any false positives. Fig. 4 shows the result of the classification on a small portion of a signal containing fall event invoked at about 24 seconds. Each dot represents the class where a window of $2 \mathrm{sec}$. of signal has been assigned. The event was classified as fall for eight consecutive windows while the alarm was activated after the third. Since the time to classify a window is $16 \mathrm{~ms}$, the total time to detect a fall event is about $316 \mathrm{~ms}$.

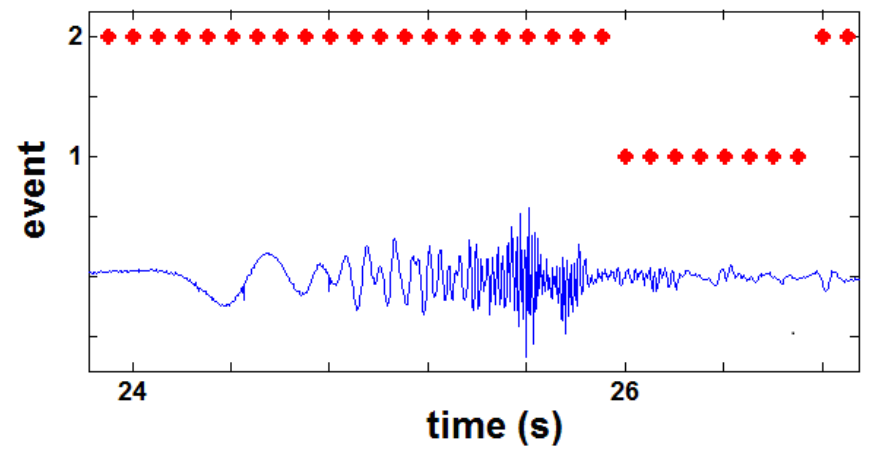

Fig. 4. Classification results of a small portion of a signal containing a fall event. In this example, the results of the classification are sent to Matlab for plotting. The fall is labelled as "1" while the normal movement as " 2 ". 


\section{Conclusion}

In this paper, an embedded healthcare system for non-invasive fall detection in indoor environment has been described. It consists of a radar sensor and a base station for data processing. The implementation of the fall detection algorithm by means of a DSP platform has been also presented. Experimental results conducted with human subjects have shown a success rate in detecting fall incidents in real-time of $100 \%$, without reporting any false positives, with a maximum delay of about $316 \mathrm{~ms}$.

The system is inline with the growing need for home health care applications and supervision technology for elderly people living at home. Next step is to integrate multiple sensors in a wireless sensor network in order to cover a whole room and also to perform in-door positioning.

Acknowledgments. This work was supported by FWO-Flanders, KU Leuven GOA Project, and the Hercules Foundation.

\section{References}

1. P. Haentjens, J. Magaziner, et al., "Excess mortality after hip fracture among older women and men," Annals of Internal Medicine, vol. 152, pp. 380-390, 2010.

2. S. R. Lord, C. Sherrington, and H. B. Menz, Falls in Older People: Risk Factors and Strategies for Prevention, Cambridge, U.K., Cambridge Univ. Press, 2007.

3. R. J. Gurley, N. Lum, M. Sande, B. Lo, and M. H. Katz, "Persons found in their homes helpless or dead," N Engl J Med., no. 334, pp. 1710-1716, 1996.

4. C. G. Moran, R. T. Wenn, M. Sikand, and A. M. Taylor, "Early mortality after hip fracture: is delay before surgery important," J. of Bone and Joint Surgery, no. 87, pp. 483-489, 2005.

5. M. Yu, S. M. Naqvi, and J. Chambers, "A robust fall detection system for elderly in a smart room," International Conference on Acoustics Speech and Signal Processing, pp. 1666 1669, Dallas, USA, March 2010.

6. Y. Zigel, D. Litvak, and I. Gannot, "A method for automatic fall detection of elderly people using floor vibrations and sound - Proof of concept on human mimicking doll falls," Transactions on Biomedical Engineering, vol. 56, no. 12, pp. 2858-2867, December 2009.

7. M. Mercuri, D. Schreurs, and P. Leroux, "Optimised waveform design for radar sensor aimed at contactless health monitoring," IET Electronic Letters, vol. 48, no. 20, pp. 12551257, Sep. 2012.

8. M. Mercuri, P. Soh, G. Pandey, P. Karsmakers, G.A.E. Vandenbosch, P. Leroux, D. Schreurs, "Analysis of an indoor biomedical radar-based system for health monitoring", IEEE Trans. Microw. Theory Tech., vol. 61, no. 5, pp. 2061-2068, May 2013.

9. P. Karsmakers, T. Croonenborghs, M. Mercuri, D. Schreurs, and P. Leroux, "Automatic indoor fall detection based on microwave radar measurements," in Proc. Eur. Radar Conf., Amsterdam, The Netherlands, Oct. 31-Nov. 2, 2012, pp. 202-205. 